\title{
28 Research Square \\ Low-grade fibromyxoid sarcoma in inguinal region:a case report and literature review
}

Lei Wang

Department of Gastrointestinal, The Third Affiliated Hospital of Soochow University

Maoling Yuan

Changzhou First People's Hospital

Feng Zhang

Changzhou First People's Hospital

Haitao Wang

Changzhou First People's Hospital

Qicheng Lu (D 326237966@qq.com)

Yong Jiang

Changzhou First People's Hospital

\section{Case report}

Keywords: Low-grade fibromyxoid sarcoma, Inguen, Inguinal mass

Posted Date: June 22nd, 2020

DOI: https://doi.org/10.21203/rs.3.rs-36119/v1

License: (1) (1) This work is licensed under a Creative Commons Attribution 4.0 International License.

Read Full License 


\section{Abstract}

Background: Low-grade fibromyxoid sarcoma (LGFMS) is a rare fibrous tumor in soft tissues.

Case presentation: A 25-year-old patient was admitted to our hospital for "more than two years with a mass in the left inguinal region". During the operation, rapid pathological examination and frozen section microscopy supported that the mass may be a low-grade mesenchymal tumor. Therefore, the surrounding tissues were resected correspondingly, Pathological diagnosis: low-grade fibromyxoid sarcoma in the inguinal region.

Conclusions: Combined with literature analysis, the diagnosis of LGFMS mainly depends on pathological diagnosis, and surgical resection is the most effective treatment.

\section{Background}

Low-grade fibromyxoid sarcoma (LGFMS) is a rare fibrous tumor in soft tissues. The main clinical manifestation shows a painless mass with slow growth located in the deep soft tissue, and a small part are located in the subcutaneous region. This report focuses on the importance of correct pre-and/or intraoperative histological diagnosis in order to offer the best therapeutic choice with the diagnosis confirmed postoperatively.

\section{Case Presentation}

The male patients aged 25 years old was accepted in our hospital. More than two years ago, the patient inadvertently observed a left inguinal mass, but the mass was ignored because of its walnut-like size. However, the mass size was gradually increased, and the mass could not go back into the abdominal cavity when lying on the bed or after pressing the mass. In addition, he felt uncomfortable, but local swelling and ulceration of the skin, fever and obvious abdominal pain distension were not observed. Thus, the patient was admitted to the hospital with "left inguinal mass". Specialist examination: there was unrecoverable mass in the left inguinal region; the local skin was not red; the mass was about $6 \mathrm{~cm}$ $\times 5 \mathrm{~cm}$ and soft; no swelling and no ulceration was observed; poor mobility was shown; at the supine position, the mass could not return into abdominal cavity; bilateral testes were at the right positions. Auxiliary examination: the B-scan ultrasonography showed that inside the left medial inguinal abdominal wall the substantial hypoechogenicity was detected as $68 \mathrm{~mm} * 38 \mathrm{~mm}$, with clear boundary and intact envelop, indicating a mass on the body skin; CDF1 demonstrated the dendritic blood flow signal of RI0.6, suggesting that the left medial inguinal abdominal wall exhibited a substantial mass with abundant blood supply. Pelvic MRI images (Fig. 1-4) revealed that patchy abnormal signal shadow was observed in the subcutaneous soft tissue of the left inguinal region, recognized as long T1 and T2 signals; the edges were fuzzy with a range of about $25 \mathrm{~mm} \times 15 \mathrm{~mm} \times 36 \mathrm{~mm}$; after enhancement, the lesion was obviously enhanced, but the edge was not so clear. Diagnosis: abnormal signal of subcutaneous soft tissue in the left inguinal region indicated inflammation and seminal vesicle adenitis was not excluded. 
No obvious abnormality was illustrated for the other examinations. On Feb 9, 2012, surgery was performed under epidural anesthesia. During the operation, with the mass as the center, an oblique incision about $5 \mathrm{~cm}$ was cut parallel to the inguinal region, and after incising the skin, the mass was observed under the skin, with a size of about $5 \mathrm{~cm} * 6 \mathrm{~cm}$. The mass was substantial without seepage, and exhibited an intact envelop and clear boundary. Along the mass edge, blunt and sharp dissection was performed, followed by electric coagulation hemostasis, and then the mass was completely resected. Simultaneously, during the operation, rapid pathological examination and frozen section microscopy supported that the mass may be a low-grade mesenchymal tumor. Therefore, it was recommended to clinically appropriately expand the resection along the surrounding tissues of the tumor, and the immunohistochemical results would be analyzed. Then the surrounding tissues were resected correspondingly, and the wound was sutured without active bleeding.

The postoperative pathological report was described as follows. The mass was $6 \mathrm{~cm} \times 5.5 \mathrm{~cm} \times 3 \mathrm{~cm}$ in size with an envelope, the cut surface was pale yellow and gray, the mass was soft, and the lesion area was mucus gelatinous. The microscopy images (Fig. 5-6) exhibited that the tumor cells were of uniform size with spindle- or short-spindle-like shapes, and the cells showed stellate shapes in the mucus-like area; the nuclei were round or oval, and hyperchromatic; the cytoplasm was slightly pink; the spindle cell area had vortex-like or linear arrangement; dendritic vessels were also observed. Immunohistochemistry results: Vimentin (+++), Desmin (-), NSE (-), S100 (-), SMA (-), CK (-) and CD34 (-). Pathological diagnosis: low-grade fibromyxoid sarcoma in the inguinal region. The patient recovered and was discharged from the hospital 5 days after the operation. No recurrence was observed during the 7-year follow-up.

\section{Discussion And Conclusion}

Low-grade fibromyxoid sarcoma (LGFMS) is a rare fibrous tumor in soft tissues. Evans ${ }^{[1]}$ reported two cases for the first time. It has been proposed that LGFMS, as a kind of independent soft tissue tumor, usually occurs among the young and middle-aged adults (14 65 years); its incidence for males is higher than females; it mainly occurs in distal limbs and fascia of the body, and is also observed in the shoulder, neck, armpits, hips, neighboring of anus, small mesentery and omentum; the main clinical manifestation shows a painless mass with slow growth located in the deep soft tissue, and a small part are located in the subcutaneous region; nerve pain can also be resulted from the compression of the nerve by large mass. LGFMS can be divided into two types: typical LGFMS as diagnosed with initially and special one as hyalinizing spindle cell tumor with giant rosettes (HSCTGR) accompanied by giant chrysanthemumlike mass. In 1997, Lane ${ }^{[2]}$ first described HSCTGR and considered it as a special subtype of LGFMS, which have been further supported by many subsequent studies ${ }^{[3]}$. There are primary HSCTGR with LGFMS metastasis, and primary LGFMS with HSCTGR metastasis. It has been reported that both HSCTGR and LGFMS are highly differentiated sarcomas with metastatic potential, which indicates that LGFMS and HSCTGR share a common pedigree. That study also systematically analyzed the pathogenesis, tumor characteristics and biological behaviors of LGFMS and HSCTGR ${ }^{[3]}$. 
The final diagnosis of LGFMS mostly depends on pathological examination, and its pathological morphology is generally characterized by clear boundary, grayish white section, and local mucus gelatinous appearance. Large tumors may have cystic changes and generally have no bleeding or necrosis. The histological features include the following points: the fibromyxoid area alternates with the spindle tumor cell area, the tumor nucleus can be slightly heteromorphic, the spindle cell area is vortexlike or palisade-like arranged, and the mitosis is not common. Immunohistochemistry results showed that LGFMS only expressed vimentin; in the process of myofibroblast differentiation, SMA was occasionally positive; Desmin, S-100, CK, EMA and CD34 were not expressed. In the aspect of molecular biology, chromosomal translocations are found in nearly all patients with LGFMS. The t(7;16)(q32-34; p11) chromosomal translocation and the resulting fusion of FUS RNA binding protein (FUS)-cAMP responsive element binding protein 3-like 2 (CREB3L2) is the most frequently seen (75-95\% of patients with LGFMS), whilst the FUS-CREB3L1 fusion gene is present in approximately $5 \%$ of cases of LGFMS ${ }^{[4,5]}$. Both the CREB3L2 and CREB3L1 genes encode transcription factors ${ }^{[6]}$. A single-centre retrospective review of 14 patients with LGFMS found no association between the presence of the FUS-CREB3L2 fusion gene and local recurrence or metastatic disease ${ }^{[4]}$.

At present, there is no specific drug treatment for LGFMS, and surgical resection is the only way for the treatment. LGFMS progresses slowly and the mass can last for several years. However, local recurrence can occur once or more after surgery, and the tumor can be even transferred to the lung for some patients. For patients with metastasis, surgical resection of the tumor is still the most effective method ${ }^{[4]}$, while radiotherapy and chemotherapy have no obvious benefits in reducing the recurrence, metastasis and prolonging the survival time of LGFMS patients ${ }^{[7]}$. In a case series of 36 patients with LGFMS treated with surgical resection with curative intent, 5 - and 15 -year local control rates were $83 \%$ and $79 \%$, respectively ${ }^{[5]}$. There are currently no putative prognostic biomarkers for LGFMS; however, the FUSCREB3L2 and FUS-CREB3L1 fusion genes, as well as the pathways influenced by the CREB3L2 and CREB3L1 wild-type genes, may be helpful in building a greater understanding of the pathogenesis of this rare tumour type and in developing effective therapeutic agents. For the above patients, rapid intraoperative pathology is recommended to be performed before determining the tumor nature. The tumor should be completely resected with intact envelope, and then whether the expanded resection of the surrounding tissues is needed or not will be decided according to the pathological results.

Simultaneously, the long-term follow-up is also significant for the treatment.

\section{Abbreviations}

\section{LGFMS}

Low-grade fibromyxoid sarcoma

HSCTGR

Hyalinizing spindle cell tumor with giant rosettes

\section{Declarations}


Ethics approval and consent to participate

Written and informed consent was obtained from the patient for the publication of this study; moreover, no patient health identification $(\mathrm{PHI})$ was recorded.

\section{Consent for publication}

Written informed consent was obtained from the patient for publication of this case report and any accompanying images.

\section{Availability of data and materials}

N/A.

\section{Competing interests}

The authors declare that they have no competing interests.

\section{Funding}

The study did not receive any funding by any governmental or private organization.

\section{Authors' contributions}

WL and YML performed the majority of this study and wrote the manuscript;

LQC, JY WHT and ZF provided the collection of material from the database.

All authors read and approved the final manuscript

\section{Acknowledgements}

The authors would like to thank the patient for their kind participation and cooperation in our study. 


\section{References}

1. Evans HL 1. ow-grade fibromyxoid sarcoma:a report of two metastasizing neoplasms havings a deceptively benign appearance. Am J Clin Pathol. 1987;88(5):615-9.

2. Lane KL. Shannon RJ,Weiss SW.Hyalinizing spindle cell tumor with giant rosettes: a distinctive tumor closely resembling low-grade fibromyxoid sarcoma. Am J Surg Pathol. 1997;21(12):1481-8. PMID:9414192 DOI:10.1097/00000478-199712000-00011.

3. $10.1097 / 00000478-200010000-00004$

Folpe AL, Lane KL, Paull G, Weiss SW. Low-grade fibromyxoid sarcoma and hyalinizing spindle cell tumor with giant rosettes: a clinicopathologic study of 73 cases supporting their identity and assessing the impact of high-grade areas.Am J Surg Pathol,2000,24(10):1353-1360.PMID: 11023096 DOI: 10.1097/00000478-200010000-00004.

4. $10.1155 / 2013 / 256280$

Maretty-Nielsen K, Baerentzen S, Keller J, Dyrop HB, Safwat A. Low-grade fibromyxoid sarcoma: incidence, treatment strategy of metastases, and clinical significance of the FUS Gene. Sarcoma. 2013;2013:256280. PMID: 23818812. DOI: 10.1155/2013/256280.

5. Zagars GK, Ballo MT, Pisters PWT, Pollock RE, Patel SR, Benjamin RS, Evans HL. Prognostic factors for patients with localized soft-tissue sarcoma treated with conservation surgery and radiation therapy: An analysis of 1225 patients. Cancer. 2003;10:2530-43. DOI:10.1002/cncr.11365. 2543 PMID: 12733153.

6. Mertens F, Fletcher CDM, Antonescu CR, Coindre JM, Colecchia M, Domanski HA, Downs-Kelly E, Fisher C, Goldblum JR, Guillou L, Reid R, Rosai J, Sciot R, MandahI N, Panagopoulos I. Clinicopathologic and molecular genetic characterization of low-grade fibromyxoid sarcoma, and cloning of a novel FUS/CREB3L1 fusion gene. Lab Investig. 2005;85(3):408-15.

DOI:10.1038/labinvest.3700230. PMID: 15640831.

7. Chamberlain F, Engelmann B, Al-Muderis O, Messiou C, Thway K, Miah A, Zaidi S, Constantinidou A, Benson C, Gennatas S, Jones RL. Low-grade Fibromyxoid Sarcoma: Treatment Outcomes and Efficacy of Chemotherapy. In Vivo. 2020;34(1):239-45. DOI:10.21873/invivo.11766. PMID: 31882484.

\section{Figures}




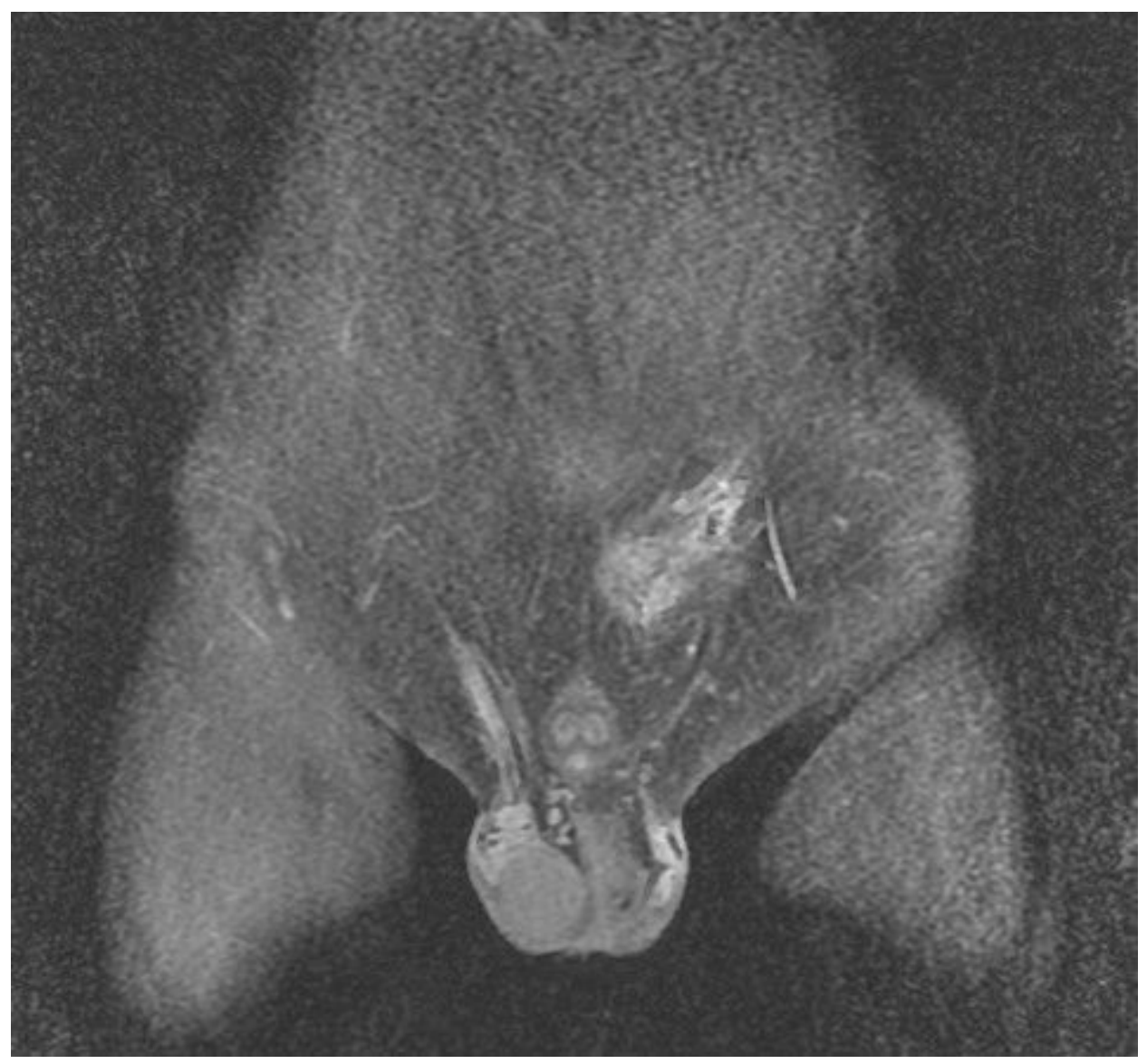

\section{Figure 1}

Pelvic MRI images (Figure 1-4) revealed that patchy abnormal signal shadow was observed in the subcutaneous soft tissue of the left inguinal region, recognized as long T1 and T2 signals; the edges were fuzzy with a range of about $25 \mathrm{~mm} \times 15 \mathrm{~mm} \times 36 \mathrm{~mm}$; after enhancement, the lesion was obviously enhanced, but the edge was not so clear. 


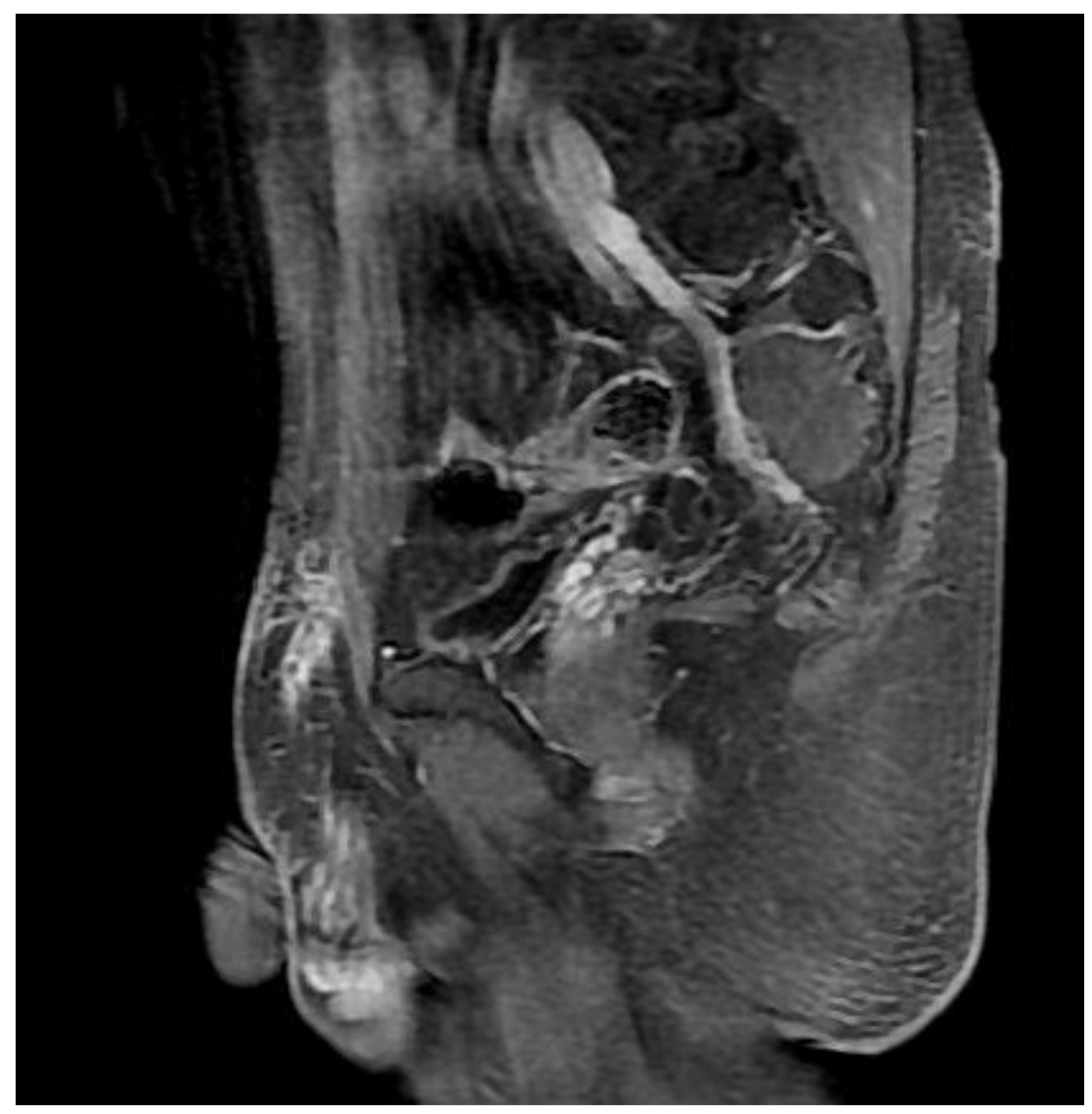

\section{Figure 2}

Pelvic MRI images (Figure 1-4) revealed that patchy abnormal signal shadow was observed in the subcutaneous soft tissue of the left inguinal region, recognized as long T1 and T2 signals; the edges were fuzzy with a range of about $25 \mathrm{~mm} \times 15 \mathrm{~mm} \times 36 \mathrm{~mm}$; after enhancement, the lesion was obviously enhanced, but the edge was not so clear. 


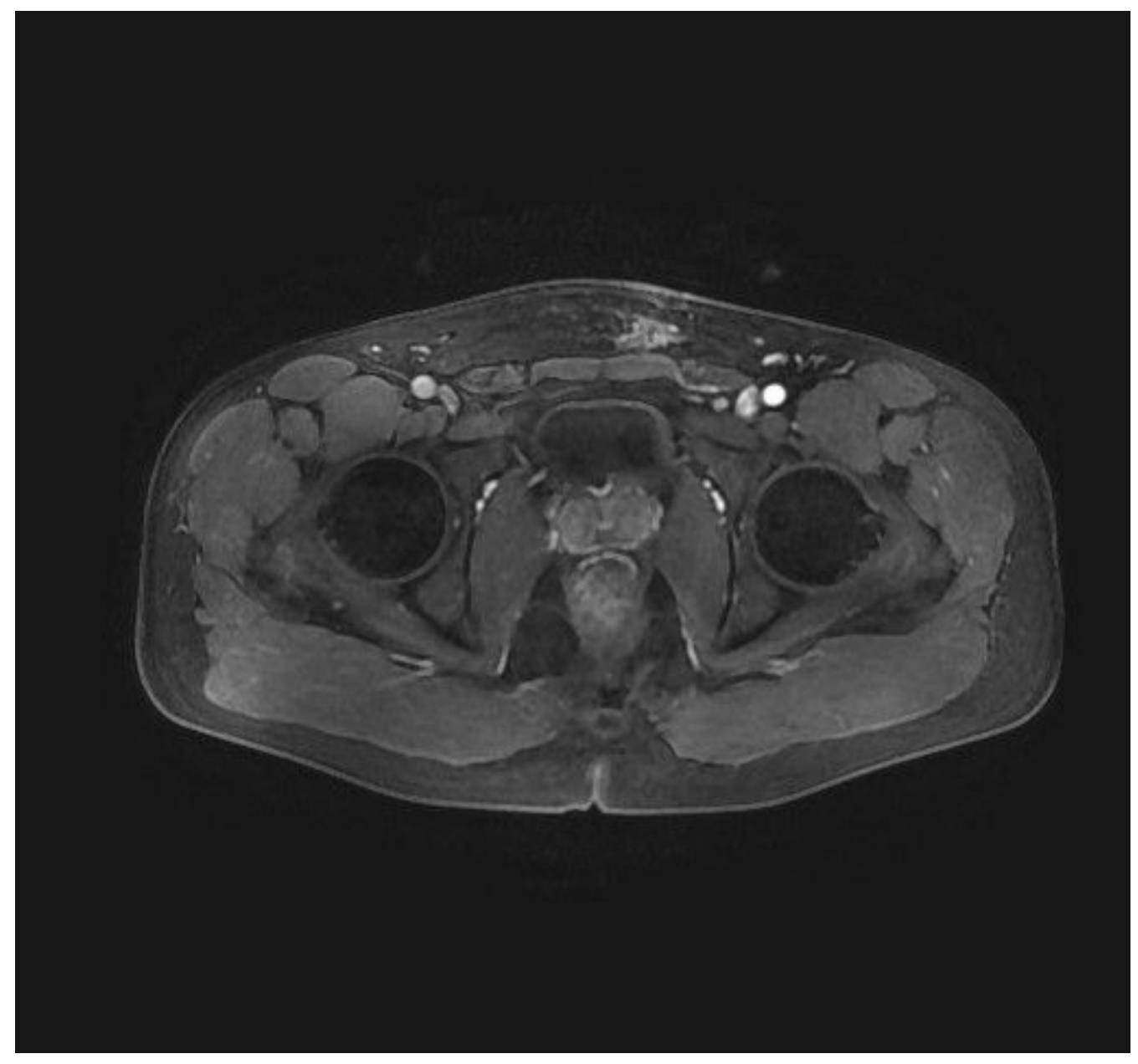

\section{Figure 3}

Pelvic MRI images (Figure 1-4) revealed that patchy abnormal signal shadow was observed in the subcutaneous soft tissue of the left inguinal region, recognized as long T1 and T2 signals; the edges were fuzzy with a range of about $25 \mathrm{~mm} \times 15 \mathrm{~mm} \times 36 \mathrm{~mm}$; after enhancement, the lesion was obviously enhanced, but the edge was not so clear. 


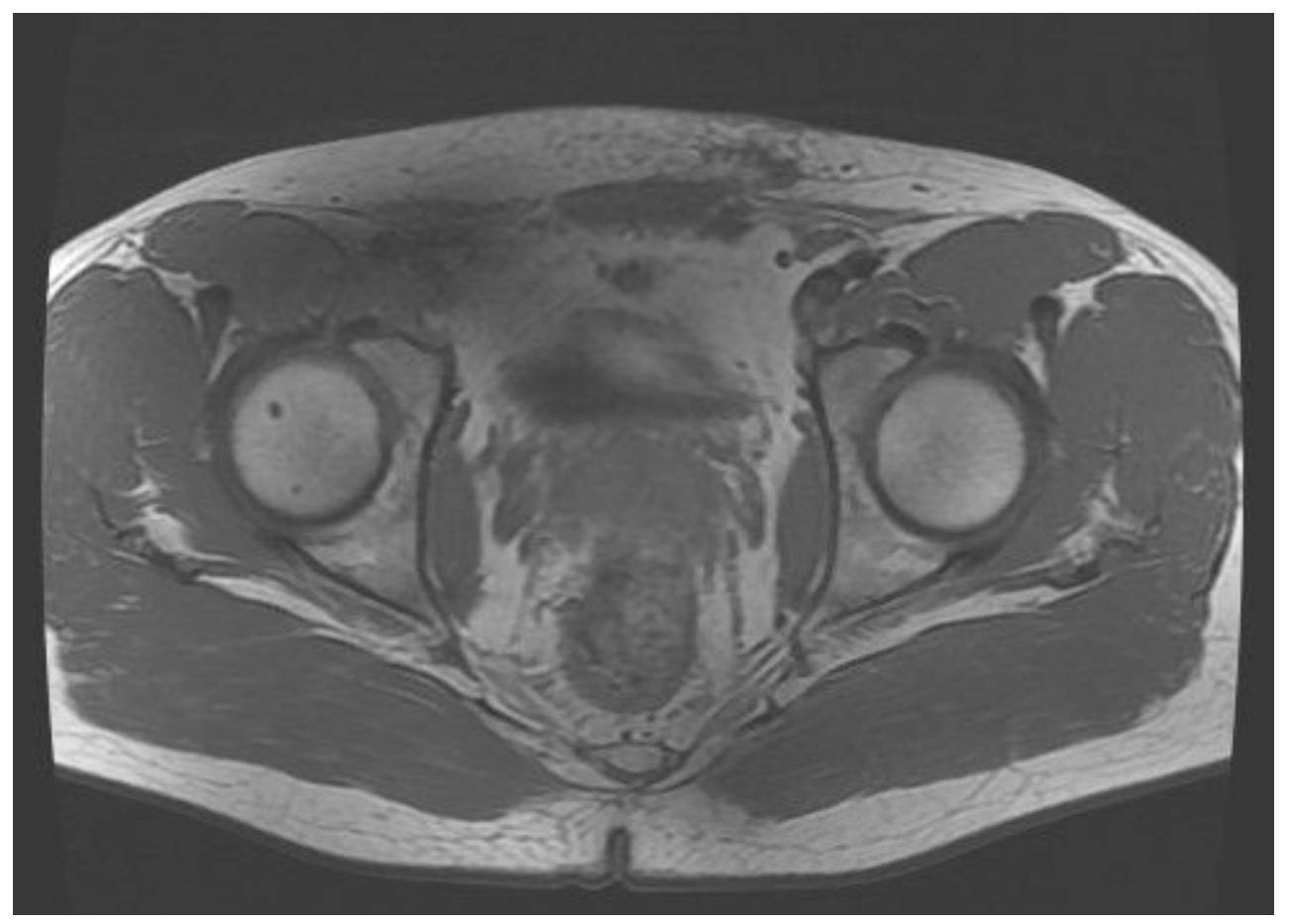

Figure 4 


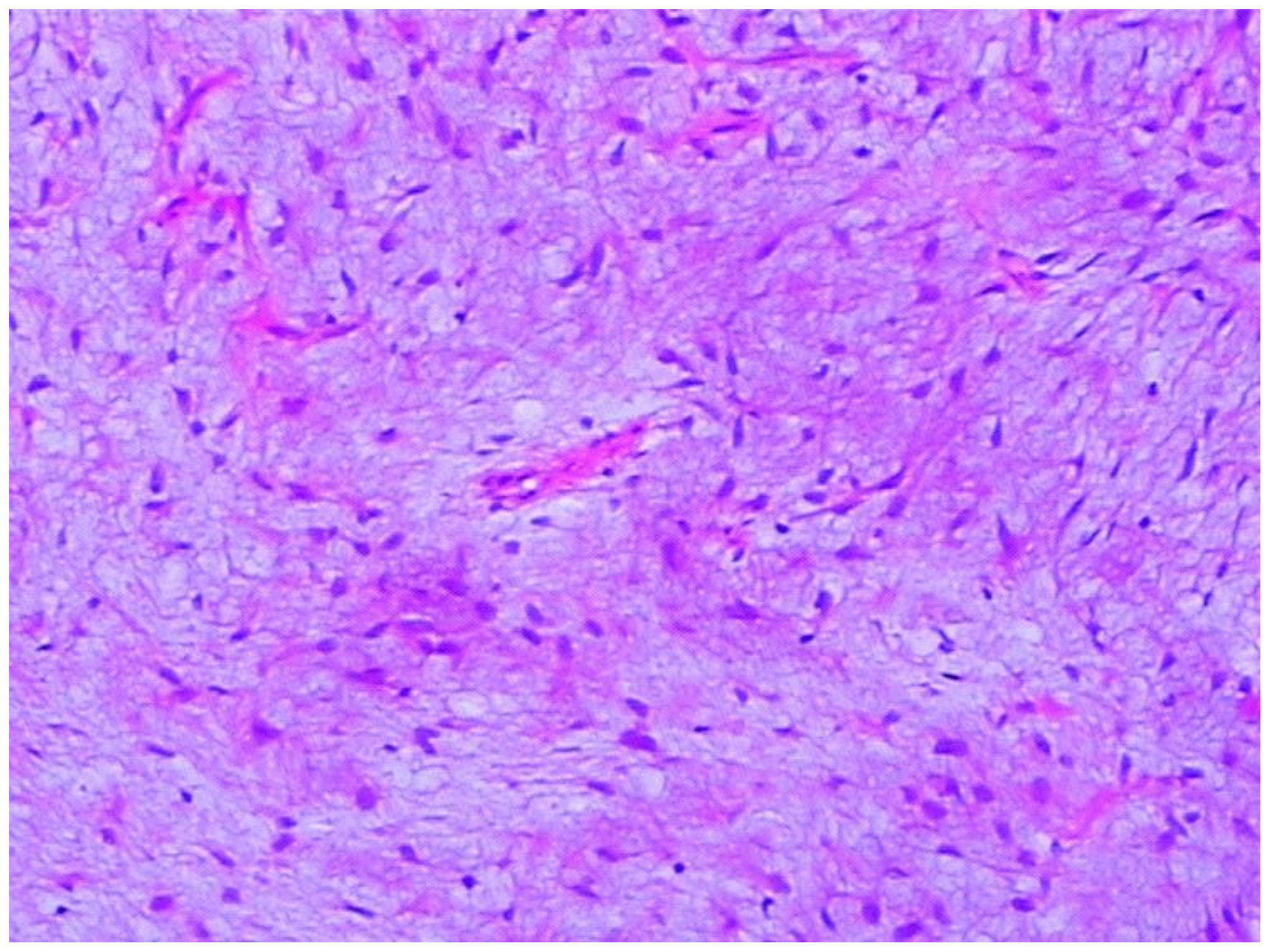

Figure 5 


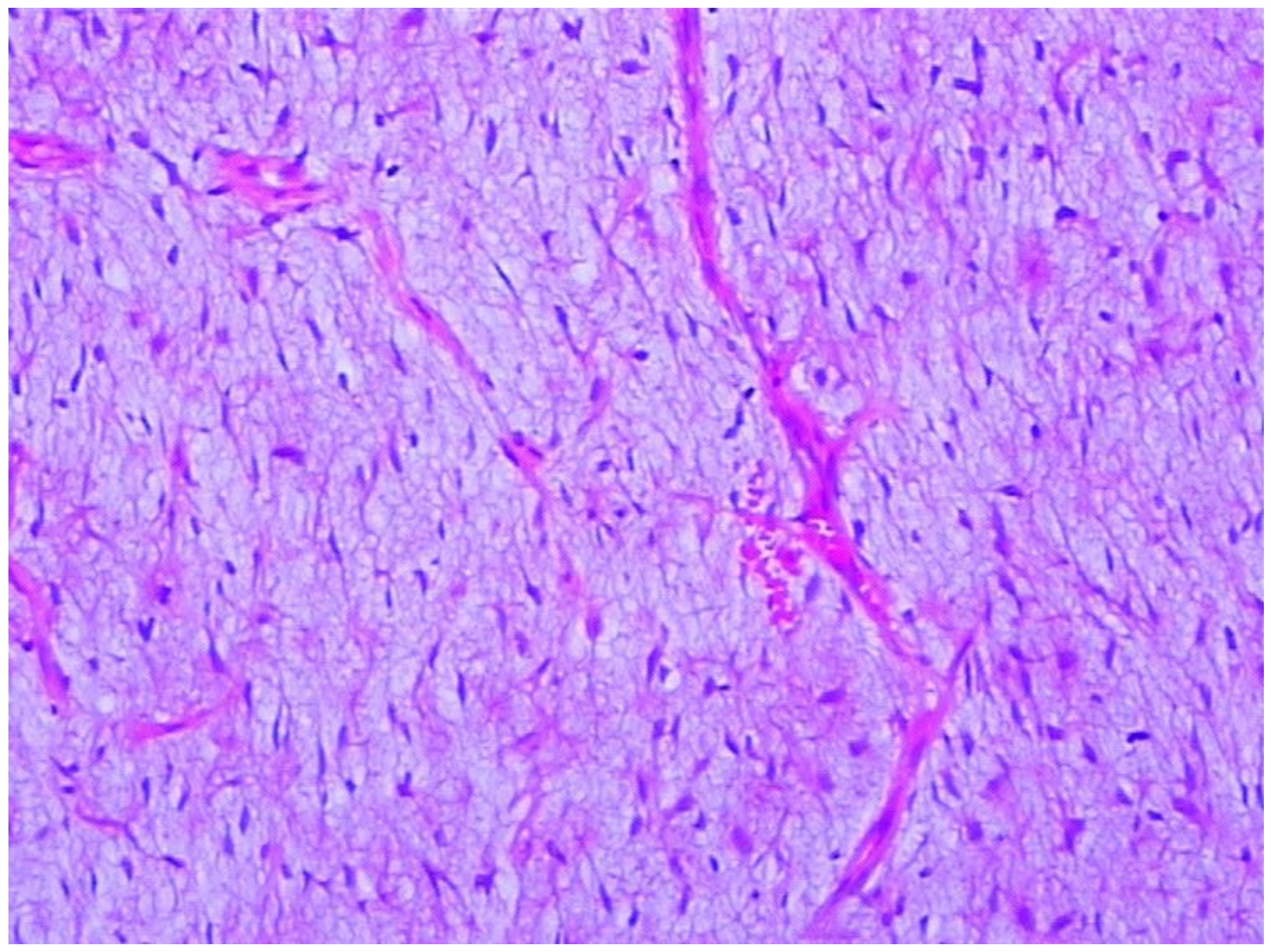

Figure 6

\section{Supplementary Files}

This is a list of supplementary files associated with this preprint. Click to download.

- renamed6bf48.doc 